\title{
DEPENDENCE OF THE INSULATOR-METAL TRANSITION IN EUO ON MAGNETIC ORDER*
}

\author{
Y. SHAPIRA and S. FONER \\ Francis Bitter National Magnet Laboratory, MIT, Cambridge, Mass. 02139, USA
}

T.B. REED

Lincoln Laboratory, MIT, Lexington, Mass. 02173, USA

and

H. BIRECKI and H.E. STANLEY

Department of PHysics, MIT, Cambridge, Mass. 02139, USA

Received 22 August 1972

\begin{abstract}
The Hall coefficient in an EuO sample which exhibited an isulator-metal transition was measured at $T \approx 4 T_{\mathrm{c}}$ in fields up to $150 \mathrm{kOe}$. The results indicate that the activation energy does not vary linearly with magnetization, contrary to the conclusion of Penney et al.
\end{abstract}

Recently Penney et al. concluded that the activation energy of insulator-metal transition in EuO varies linearly with the magnetization [1]. In this letter we present data which disagree with this conclusion.

The resistivity $\rho$ of some EuO samples decreases by many orders of magnitude between the Curie temperature $\left(T_{\mathrm{c}}=69 \mathrm{~K}\right)$ and $50 \mathrm{~K}[1,2]$. This "insulator-metal transition" is due primarily to a change in the carrier concentration $n$, although a change in the mobility $\mu$ may be present. A model which explains the insulator-metal transition was proposed by Oliver et al. [2] and was modified by the group at IBM [1] . In this model, above $\sim 50 \mathrm{~K}, \boldsymbol{n}$ is proportional to $\exp (-\Delta / k T)$, where $\Delta$ is an activation energy which depends on magnetic order. At $T \gg T_{\mathrm{c}}$, $\Delta$ assumes a constant value, $\Delta_{0}$, and $\rho \sim \exp \left(\Delta_{0} / k T\right)$.

Recently Penney et al. [1] considered two possible dependences of $\Delta$ on magnetic order: 1) $\Delta$ varies linearly with the reduced magnetization (long range order parameter) $\sigma=\langle S\rangle / S$, i.e.,

$\Delta=\Delta_{0}(1-a \sigma)$,

where $a$ is a constant;2) $\Delta$ varies linearly with the nearest-neighbor two-spin correlation function (short

\footnotetext{
* Work supported by NSF, by the Department of the Air Force, and by ONR.
}

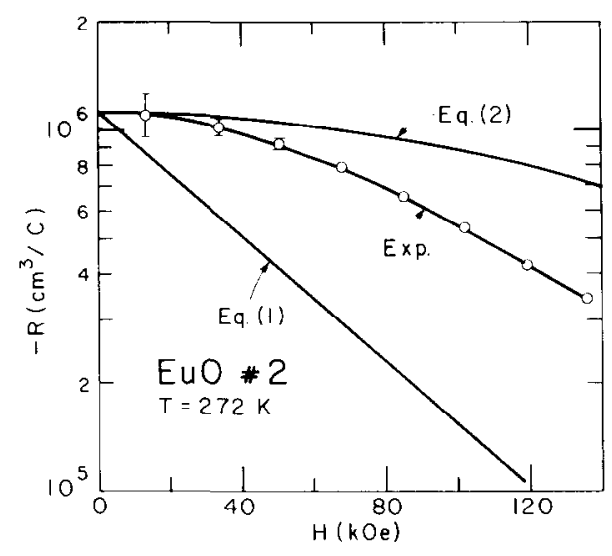

Fig. 1. $H$-dependence of the Hall coefficient at $272 \mathrm{~K}$ (semi$\log$ scale). The theoretical curves were calculated from eqs. (1) and (2) using the magnetic susceptibility of this sample, and $\Delta_{0}=0.32 \mathrm{eV}, a=1.39, b=1.5, \eta(H)-\eta(0)=1.08 \sigma^{2}$

range order parameter) $\eta=\left\langle\boldsymbol{S}_{1} \cdot \boldsymbol{S}_{2}\right\rangle / S^{2}$, i.e.,

$\Delta=\Delta_{0}(1-b \eta)$,

where $b$ is a constant. From their analysis of $\rho(T)$ at $T<T_{\mathrm{c}}$ Penney et al. concluded that $\Delta$ obeys eq. (1) but not eq. (2). However, in this analysis the possibility that $\mu$ depends on $T$ was not taken into account. In addition, agreement of the resistivity data at 
$20 \mathrm{kOe}$ with eq. (1) was obtained by assuming the largest possible demagnetizing factor, $4 \pi$. This assumption is questionable.

In the present work the dependence of $\Delta$ on magnetic order was studied by measuring the Hall coefficient $R=\mid 1 /$ ne $\mid$ near room temperature $\left(T \approx 4 T_{\mathrm{c}}\right)$ in magnetic fields up to $150 \mathrm{kOe}$. These measurements provide a meaningful test of eqs. (1) and (2) because: a) at $T \approx 4 T_{\mathrm{c}}$ these equations predict vastly different behaviors for $R$ versus $H$, and b) the analysis of the results at $T \approx 4 T_{\mathrm{c}}$ is not sensitive to mobility changes or to demagnetization corrections.

At $T \approx 4 T_{\mathrm{c}}, \sigma \approx 0.15$ at $150 \mathrm{kOe}$ and $\sigma$ is proportional to $H$ at lower fields. Molecular field theory gives $\eta=\sigma^{2}$. A better estimate of $\eta(H)$ was obtained from a calculation based on a high-temperature series expansion. Our calculation showed that although $\eta$ does not vanish at $H=0$, the $H$-induced increase in $\eta$ at $4 T_{\mathrm{c}}$ is well described by $\eta(H)-\eta(0)=1.08 \sigma^{2}$. At $150 \mathrm{kOe}, \sigma^{2} \approx 0.02 \ll \sigma$. These considerations show that eq. (1) leads to a linear dependence of $\log R(H)$ on $H$, whereas eq. (2) leads to a quadratic dependence. Resistivity data below $T_{\mathrm{c}}$ give values for $a$ and $b$, in eqs. (1) and (2), which are roughly equal. With these values for $a$ and $b$, eq. (1) predicts a much larger variation of $R$ with $H$ at $T \approx 4 T_{\mathrm{c}}$ than eq. (2), since $\eta(H)-\eta(0) \ll \sigma(H)$
Measurements were carried out on a single crystal EuO sample which exhibited a clear insulator-metal transition. For this sample the $T$-dependence of $\rho$ at $T \gg T_{\mathrm{c}}$ gave $\Delta_{0}=0.32 \mathrm{eV}$. Analysis of $\rho(T)$ below $T_{c}$, using the procedure described in [1] and assuming the validity of eq. (1), gave $\Delta_{0}=0.28 \mathrm{eV}$ and $a=1.39$. A value $b \approx 1.5$ for the coefficient in eq. (2) was estimated from the value of $\eta$ at the onset of the insulator-metal transition (where $\Delta \rightarrow 0$ ).

Experimental results for $R(H)$ versus $H$ at $272 \mathrm{~K}$ are shown in fig. 1 , together with the $H$-dependence of $R(H)$ calculated from eqs. (1) and (2) using $\Delta_{0}=$ $0.32 \mathrm{eV}$. The experimental data lie between the two calculated curves. Moreover, analysis shows that $\log [R(H) / R(0)] \sim H^{r}$ with $r=1.8$, which is between $r=1$ [eq. (1)] and $r=2$ [eq. (2)]. These conclusions remain unchanged if a value $\Delta_{0}=0.28 \mathrm{eV}$ is used instead of $0.32 \mathrm{eV}$. A similar behavior of $R(H)$ was also observed at $288 \mathrm{~K}$. We therefore conclude that near $4 T_{c}$ the dependence of $\Delta$ on magnetic order is intermediate between eq. (1) and eq. (2).

\section{References}

[1] T. Penny, M.W. Shafer and J.B. Torrance, Phys. Rev. B5 (1972) 3669 , and refererence therein.

[2] M.T. Oliver et al., Phys. Rev. BS (1972) 1078. 